\title{
Experimental Investigation of a Novel Formulation of a Cyanoacrylate-Based Adhesive for Self-Healing Concrete Technologies
}

\begin{abstract}
Cristina De Nardi ${ }^{1}$, Diane Gardner ${ }^{1}$, Giulia Cazzador ${ }^{3}$, Davide Cristofori ${ }^{2}$, Lucio Ronchin ${ }^{3}$, Andrea Vavasori ${ }^{3}$ and Tony Jefferson ${ }^{1}$

${ }^{1}$ Resilient Materials for Life (RM4L) Research Group, School of Engineering, Cardiff University, Cardiff, United Kingdom, ${ }^{2}$ Centre for Electron Microscopy "Giovanni Stevanato" and Department of Molecular Sciences and Nano Systems, Ca' Foscari University of Venice, Venice, Italy, ${ }^{3}$ Department of Molecular Sciences and Nano Systems, Ca' Foscari University of Venice, Venice, Italy
\end{abstract}

The selection of an appropriate healing agent is critical to the success of vascular and minivascular networks. In self-healing concrete technology, commercially available cyanoacrylate (CA) adhesives have been shown to produce good strength recoveries; however, their rapid curing rate and short shelf-life make them unsuitable for site application. The aim of this study was to develop a modified cyanoacrylate ( $n-C A$ ) with an extended shelf-life suitable for incorporation in a self-healing system. A series of $n$-CAs were formed from a commercial Ethyl Cyanoacrylate adhesive mixed with acrylic acid (AA) and nitro-anthraquinone (nAq) in varying ratios. When encapsulated within 3D printed mini-vascular networks (MVNs), the $\mathrm{n}$-CAs remained dormant in liquid form for up to 5 days. The contact angle between the $\mathrm{n}$-CAs and the cement mortar substrate, as measured via the sessile drop technique, decreased significantly with increasing AA content. The mechanical properties (bond strength) and the polymerization hardening of the n-CAs were evaluated over a curing period of 7-21 days, via a series of pull-off tests using cement mortar cubes. The 4:1:02 (CA: AA:nAq) $n$-CA formulation showed a significant increase in bond strength from 14 to 21 days, with a ceiling value of $2.6 \mathrm{MPa}$, while the 2:1 (CA:AA) n-CA formulation exhibited a good bond strength after 21 days (1 MPa). Nuclear Magnetic Resonance (NMR) conducted on the $\mathrm{n}$-CAs suggested the formation of several new polymeric species, whilst differential scanning calorimetry (DSC) and thermogravimetric analysis (TGA) on the pre- and post-printed MVN material confirmed no significant changes in chemistry with no evidence of polymer degradation. Considered together, the experimental results show the potential for different $\mathrm{n}-\mathrm{CA}$ formulations to act efficiently as a healing agent.

Keywords: self-healing concrete, cyanoacrylate adhesives, vascular and mini-vascular networks, 3D printing, healing agent

\section{INTRODUCTION}

Cyanoacrylate-based adhesives are commonly colourless, one-part, room-temperature curable, low viscosity liquids. These properties, in addition to the ability to cure rapidly and to form rigid thermoplastics with excellent adhesion with the substrates, make cyanoacrylates the most widely used adhesive in both industrial and structural applications (Petrie, 2007; Irving, 1990). 
A number of investigators have used cyanoacrylate-based adhesives in technologies for self-healing concrete structures ( $\mathrm{Li}$ et al., 1998; Zhang et al., 2020). Cyanoacrylate (CA) is a costeffective healing agent, with good rheological properties that allow migration to areas of damage and good penetration into the cementitious matrix (Joseph et al., 2010). Moreover, rapid curing cyanoacrylates are applicable to situations in which the healing domain (crack opening) changes over periods of hours or days. In such scenarios, the bonding processes of sloweracting agents (e.g., sodium silicate) are greatly disrupted by the moving crack surfaces, whereas the changes in the crack dimensions are minimal over the curing period of a fastacting CA. The rapidity of CA curing also enhances the potential for repeated healing events (Ebnesajjad, 2009; Selvarajoo et al., 2020). Cyanoacrylate-based adhesives in self-healing cementitious materials have been explored by several researchers (Li et al., 1998; Joseph et al., 2011; Sun et al., 2011). In most investigations, the adhesive was stored inside hollow glass tubular capsules. In such systems, the agent is released when a crack (or multiple cracks) in the cementitious matrix crosses and breaks the brittle tube. Pioneering work carried out by Dry in 1994 (Dry, 1994), showed that the CA stored within the glass tubes was successfully released into the damaged concrete beams, sealing the crack and preventing its reopening in subsequent tests. Li et al. (1998) also highlighted a regain of flexural stiffness in beams containing hollow glass fibers filled with CA after an initial cycle of loading. Joseph et al. (2010) investigated the influence of adhesive-filled glass reservoirs within reinforced mortar beams. The study underlined that CA was successfully delivered into the crack and transported toward the crack tip by capillary forces, which also resulted in good penetration into the whole micro-cracked region. Moreover, an apparent enhancement of the post-healed beam properties, when compared with the virgin properties, was observed.

However, the inherent fragility of glass tubes limits their ability to survive the concrete mixing process and the increased time needed to place the glass tubes within the concrete formwork -prior to casting-is incompatible with large-scale application (Xue et al., 2020). To overcome some of these challenges, glass tubes have been replaced with channels, which mimic the vascular networks carrying clotting and nutrients agents in the human body. Davies et al. (Davies et al., 2021) formed 1D and 2D vascular networks using a series of linear interconnecting hollow channels. The supply and the delivery of the agents (i.e. sodium silicate (SS) and CA) and was improved by pressurizing the healing agent within the network. It was noted that the SS proved easier to handle and supply into the vascular network, whilst CA offered greater strength recovery (up to $90 \%$ ) in a relatively short timescale (Davies et al., 2015). Selvarajoo et al. (2020), Klemarczyk and Guthrie (2010) showed that the degree and rate of healing depend significantly on the crack opening and its rate during the healing period (for crack openings in the range $0.1-0.3 \mathrm{~mm}$ ). Moreover, the rate of CA curing, from the front adjacent to the substrate wall, gradually decreases over time due to the fact that the cured CA forms an increasingly wide barrier to the source of hydroxide ions, which are needed for the curing reactions. Gardner et al. (Gardner et al., 2012) studied the capillary flow of CA in cracks in cementitious materials and showed that CA's viscosity remained unchanged over a time period of $15 \mathrm{~min}$ for the capillary diameters used. Gardner et al. also developed a modified form of the Lucas-Washburn equation to simulate the capillary flow behavior of the healing agent and considered the stick-slip behavior of the meniscus and frictional dissipation at the meniscus wall boundary. The research was later extended to explore the meniscus characteristics of CA and ggbs in suspension on different substrates (Gardner et al., 2017). In particular, the capillary meniscus contact angles of both healing agents on different substrates (i.e., glass, saturated concrete and unsaturated concrete) was evaluated. Furthermore, it was shown that the flow behavior of both healing agents in a closed channel, in the liquid state, conformed closely to Poiseuille's law. Additionally, it was found that CA's viscosity increased over the 40-min period that it was exposed to a cementitious environment.

The internal environment in a concrete matrix may be considered compatible with CA's curing requirements. CAs are acid solutions that are (to a degree) neutralized when in contact with the alkaline concrete environment, which results in accelerated curing. This can be beneficial when rapid healing is required; however, if the curing time is too quick, the dispersion of the healing agent within the matrix might be insufficient (Joseph et al., 2007). Moreover, the hazards of handling CA adhesives due to their rapid bonding to skin coupled with their high toxicity and relatively short shelf-life make CA extremely challenging for site application. Generally, cyanoacrylates have a shelf life of 6-12 months after which aging may cause a slower rate of polymerization and low peel strength along with poor solvent and temperature resistance (Klemarczyk, 2002). The extreme reactivity of the CA monomer also makes it difficult to encapsulate (Davies et al., 2015).

Akin to capsule-based self-healing technologies, minivascular networks (MVNs) require the healing agent to be kept in a dormant state until a crack occurs in the cementitious matrix. At such time, one or more ligaments of the MVNs breaks, releasing the healing agent and thereby healing the crack. The success of the healing system is strongly related to its specifications, such as the viscosity, encapsulated shelf-life, the mechanical properties of the cured/polymerized healing agent coupled with the stiffness of the MVNs (De Nardi et al., 2020).

Highly reactive cyanoacrylate ester monomers, such as ethyl cyanoacrylate, polymerize through anionic polymerization at room temperature, with trace amounts of water or amines as catalysts. Generally, surfaces contain sufficient absorbed water to catalyze the polymerization. The chemical reaction progresses very quickly and results in rapid bonding (within seconds) while the complete reaction might take from $30 \mathrm{~s}$ (for rubber) to 1-2 min (metals and plastics) (Petrie, 2007). Investigations on the encapsulation of CA in $3 \mathrm{D}$ printed polylactide acid (PLA) tetrahedral mini vascular networks (TETs) by De Nardi et al. (De Nardi et al., 2020) showed a tendency for the CA to prematurely cure within the MVN units (after $72 \mathrm{~h}$ ). It is believed that the 
presence of the hydroxyl group in the PLA molecular structure initiated the curing process and caused a change in CA's viscosity. This resulted in little or no release of the adhesive when the MVNs were broken 3 days or more after encapsulation (De Nardi et al., 2020).

The aim of the work reported in this paper was to develop a modified CA with enhanced performance that satisfies the healing agent requirements for concrete technologies; namely, 1) the ability to remain liquid and chemically stable for a long time; 2) the ability to prevent any chemical interactions with the carriers that can change their mechanical properties; 3 ) the ability to react with the cementitious matrix - when released-and to form compatible compounds.

Different formulations containing 2-ethyl cyanoacrylate (CA) (a commercial monomer), acrylic acid (AA) and nitroanthraquinone (nAq) were prepared by mixing the components CA:AA:nAq in the ratios $\mathrm{x}: 1: 0.02$, where $\mathrm{x}$ is in the range from 5 to 1 . The cyanoacrylate monomer was selected as a basic healing agent for its flow properties coupled with good bond strength as well as good aging and weathering properties (Kotzev et al., 1981; Klemarczyk and Guthrie, 2010). When a crack appears in the cementitious matrix, the adhesive is thereby intended to be released into the crack plain, cure, and thus heal the original crack through bonding of the crack faces. This healing process has also been studied by other authors ( $\mathrm{Li}$ et al., 1998; Hirozo et al., 2000; Nishiwaki et al., 2014).

The development of bond strengths at different curing durations (7-21 day) were evaluated and compared with those produced with the commercial 2-ethyl cyanoacrylate control. In addition, the degree of wetting was evaluated using contact angle measurements.

The paper is structured as follows

- Modified Ethyl Cyanoacrylate (n-CA) provides an overview of a series of investigations concerning the chemical formulation of modified cyanoacrylate (n-CA). Several candidate formulations were studied that were selected on the basis of their ability to act as retarder of the starting CA monomer and their negligible/low reactivity. Different proportions of the chosen components were considered using longevity tests conducted on $3 \mathrm{D}$ printed PLA polymer units.

- Experimental Materials and Methods describes a set of experiments which determined the physical-chemical and mechanical properties of four n-CA formulations. The bond strength to cementitious substrates and the degree of cure over time (7-21 days) were evaluated by means of a pull-off test. The interaction of the $\mathrm{n}$-CA formulations with a cementitious surface was evaluated using the sessile drop technique (contact angle measurements).

- Section 4 reports a series of tests conducted to characterize the PLA polymer used to manufacture the mini vascular network units. DSC and TGA analysis were performed on fragments collected from the filament roll before the printing phase and from the TETs immediately after printing. The results of the NMR analysis carried out on a selected formulation are also presented.

\section{MODIFIED ETHYL CYANOACRYLATE (N-CA)}

In order to meet the encapsulation process requirements, such as the ability to remain liquid for a long period of time coupled with the ability to prevent any chemical interactions with the carriers, the design of the n-CA formulation was primarily focused on reducing the time of curing, introducing components (solvents) that are chemically compatible with the PLA while maintaining good bond properties with the cementitious substrate.

\section{(n-CA) Formulation}

Among the various solvents, selected organic acids are good candidates as polymerization retarders since they protonate water and all bases eventually within the reaction solution, which start the polymerization process. It is noteworthy that a small quantity of a base can initiate the CA polymerization, moreover the ionic polymerization of CA may also take place via radical initiation. In fact, hydroquinone is present in commercial CA to stabilize such a reagent.

The first prerequisite of the solvent is the ability to solubilize the cyanoacrylate whilst being inert toward PLA. Table 1 reports selected examples concerning the solubility of CA and PLA in some solvents (at $25^{\circ} \mathrm{C}$ ). The solubility test was prolonged for 20hrs of treatment.

Three solvents fulfilled the preliminary requirements, i.e. the ability to act as solvent of the starting 2-ethyl cyanoacrylate monomer but not of the PLA: styrene, acrylic acid and acetic acid.

Styrene is widely recognized as a mutagen and thus potentially carcinogenic (Quartarone et al., 2014), as such its inclusion in the formulations is not recommended, although it will be included here for completeness. Due to concerns regarding the interaction of acetic acid with the highly alkaline nature of the hydration reactions of the cementitious matrix, the only remaining candidate solvent is the acrylic acid.

Several formulations have been studied, by adding the chosen solvents in different proportions and in some cases with the addition of benzoylhydroperoxide as a standard initiator for radical polymerization (Odian, 2004), as reported in Table 2.

All the formulations containing the acrylic acid, without the addition of the initiator, showed a good polymerization rate, i. e., $<7$ days. The reaction takes place at room temperature $\left(25^{\circ} \mathrm{C}\right)$ and it is activated without the need for mechanical agitation, thermal or radiation treatment. When the formulation containing the initiator (benzoylhydroperoxide) is encapsulated into the $3 \mathrm{D}$ printed units, the cyanoacrylate monomer is inherently less stable. The addition of an initiator such as the benzoylhydroperoxide into the concrete mix design would introduce a light toxicity into the whole matrix. Moreover, because of their reactivity, the long-term stability of hydroperoxides is not guaranteed in a highly alkaline medium such as a cementitious matrix. The same can be said of polymerization initiators in general. However, the use of the radical initiator allows a comparison with a polymer whose monomer conversion is optimal under the condition investigated. The detailed description and impact of the 
TABLE 1 | Solubility test: Ethyl Cyanoacrylate, Polylactide acid.

Solubilization test outcome

Ethyl cyanoacrylate (CA)

Polylactide acid (PLA)

Dichloromethane

Diethyl ether

Styrene

Acrylic acid

Ethyl acrylate

Chloroform

Acetic acid

Trifluoroacetic acid

Acetic anhydride

Trifluoroacetic anhydride

$Y$

$\begin{array}{lr}Y & Y \\ N & N \\ Y & N \\ Y & N \\ N & N \\ Y & N \\ Y & Y \\ Y & N \\ N & Y \\ N & Y \\ Y & N\end{array}$

TABLE 2 | Polymerization time and aspect of different formulations.

\begin{tabular}{|c|c|c|c|c|c|c|}
\hline \multirow[t]{2}{*}{ Formulation } & \multirow{2}{*}{$\begin{array}{c}\text { Ethyl cyanoacrylate } \\
\text { (g) }\end{array}$} & \multicolumn{2}{|c|}{ Solvent } & \multirow[t]{2}{*}{ Initiator } & \multirow{2}{*}{$\begin{array}{l}\text { Polymerization time } \\
\text { (days) }\end{array}$} & \multirow{2}{*}{$\begin{array}{c}\text { Appearance afte } \\
\text { polymerization }\end{array}$} \\
\hline & & Type & Weight (g) & & & \\
\hline 1 & 2.00 & Acrylic acid & 6.00 & $N$ & 7 & Viscous gel \\
\hline 2 & 3.00 & Acrylic acid & 6.00 & $\mathrm{~N}$ & 4 & Vitreous solid \\
\hline 3 & 6.00 & Acrylic acid & 6.00 & $\mathrm{~N}$ & 4 & Vitreous solid \\
\hline 3 & 3.00 & Acrylic acid & 6.00 & $Y$ & 2 & Vitreous solid \\
\hline 4 & 2.00 & Acrylic acid & 6.00 & Y & 5 & Vitreous solid \\
\hline 5 & 2.00 & Styrene & 6.00 & $Y$ & 5 & Viscous gel \\
\hline 6 & 2.00 & Styrene & 6.00 & $\mathrm{~N}$ & 5 & Viscous gel \\
\hline
\end{tabular}

TABLE 3 | Colored n-CA composition.

\begin{tabular}{|c|c|c|c|c|}
\hline Formulation & CA/AA (g) & \multicolumn{2}{|c|}{ Coloring compound (g) } & Polymerization rate (days) \\
\hline 1 & $3 / 3$ & 1-Nitro-anthraquinone & 0.06 & $>28$ \\
\hline 2 & $3 / 3$ & 1-Nitroso-2-naphthol & 0.06 & $<1$ \\
\hline 3 & $6 / 6$ & - & - & 4 \\
\hline
\end{tabular}

inclusion of the initiator into the concrete mix design are the subject of future research.

\section{n-CA Coloring Compounds}

The formulation containing acrylic acid is a clear and colourless fluid, similar to commercial CA adhesives, but with low odor and lower bleaching than the original formulation. This presents a particular challenge when handling the adhesive, with respect to the visibility of the adhesive during its manual injection into clear PLA printed MVNs. In order to make the $\mathrm{n}$-CA adhesive readily visible to the human eye when the composition is spread in thin films, two types of coloring compounds were tested: 1-nitroanthraquinone (nAq) and 1-nitroso-2-naphthol as reported in Table 3.

As colorants in general, both the coloring compound candidates, aside from their tracer behavior, are also light activated compounds which may act as light sensitizers and thus able to modify the amount of radicals during the polymerization. It appears that the addition of 1-nitro-anthraquinone greatly retards the polymerization rate, whilst 1-nitroso-2-naphthol acts to accelerate the polymerization rate. Such behavior agrees with the ability of quinones to act as radical scavengers, whereas nitroso compounds may be the source of radicals by the formation of azo and azozy species (Quartarone et al., 2014).

The mixture containing CA/AA colored by 1-Nitroanthraquinone, showed a significant inhibition of the spontaneous polymerization, and the compound maintained the same practical flow capabilities for 28 days, after which time the increased viscosity of the compound, caused by the polymerization reactions, impedes the release of the compound from the PLA capsules. This suggests the formulation of n-CA might be encapsulated in PLA networks with good stability.

\section{n-CA Longevity Within 3D Printed Polylactide Acid Capsules}

The choice of the n-CA formulation for any specific application (e.g., vascular networks, mini-vascular networks, capsules) will depend on the anticipated ability to remain chemically inert when held in contact with the material of the 3D printed units, along with the target time of healing. Since 
TABLE 4 | Longevity test of n-CA adhesives in clear PLA.

\begin{tabular}{|c|c|c|c|c|c|c|c|c|c|c|c|c|c|}
\hline & & \multicolumn{12}{|c|}{ CA:AC: NAq ratio } \\
\hline & & \multicolumn{4}{|c|}{ 1:1:0.02 } & \multicolumn{4}{|c|}{ 2:1:0.02 } & \multicolumn{4}{|c|}{ 1:2:0.02 } \\
\hline & & 1 day & 3 days & 5 days & 7 days & 1 day & 3 days & 5 days & 7 days & 1 day & 3 days & 5 days & 7 days \\
\hline \multirow[t]{3}{*}{ Clear PLA } & PC24 & I & $\mathrm{s} / \mathrm{l}$ & $\mathrm{s} / \mathrm{l}$ & $\mathrm{s}$ & 1 & $\mathrm{~s} / \mathrm{l}$ & $\mathrm{s} / \mathrm{l}$ & s & 1 & 1 & $\mathrm{~s} / \mathrm{l}$ & s \\
\hline & PC60 & 1 & 1 & $\mathrm{~s} / \mathrm{l}$ & s & 1 & 1 & $\mathrm{~s} / \mathrm{l}$ & $\mathrm{s} / \mathrm{l}$ & 1 & I & 1 & $\mathrm{~s} / \mathrm{l}$ \\
\hline & PC90 & I & I & $\mathrm{s} / \mathrm{l}$ & s & 1 & $\mathrm{~s} / \mathrm{l}$ & $\mathrm{s} / \mathrm{l}$ & $\mathrm{s}$ & I & 1 & $\mathrm{~s} / \mathrm{l}$ & $\mathrm{s}$ \\
\hline
\end{tabular}

the mini-vascular networks/capsules must preserve the healing agent until the appearance of cracks, the compatibility with a common 3D printing polymer (i.e., clear PLA) was investigated. A number of viability tests were performed on cylindrical capsules (inner diameter $=4 \mathrm{~mm}$, length $40 \mathrm{~mm}$, double wall thickness $0.25 \times 2 \mathrm{~mm}$ ) printed from clear PLA filament ( $1.75 \mathrm{~mm}$ in diameter) provided by Innofil 3D-BASF. Samples were filled with three different CA agents: (a) Procure PC24, of medium viscosity (100 cPs); (b) Procure PC60, of medium viscosity ( $80-120 \mathrm{cPs}$ ); (c) Procure PC90, of low to medium viscosity (34-44 cPs). Samples were then sealed with silicone and broken at $24 \mathrm{~h}$ intervals to determine the physical condition of the formulation. In order to minimize the contact of the CA with moisture sources (from the external environment) all the capsules were coated with a thin layer of wax. The results of CA longevity tests are summarized in Table 4, where the state of CA is classified as liquid (L); partially solid with a liquid center and solid annulus (s/L); and solid in all tubes (s).

Formulations containing PC60 remained in a liquid state for longer than the other CA candidates, providing evidence of an ability to remain liquid after 3 days in all the formulations considered; however, commencing from the fifth day, the curing process of the adhesive mixture containing this CA seemed to be initiated and the viscosity of mixture at the end of the testing time was greater than that at the beginning. Moreover, no significant alterations of the PLA were visible after three days. This implies that $3 \mathrm{D}$ printed MVNs using clear PLA and filled with formulations containing PC60 have the potential to survive within the concrete matrix for at least three days, before possible reactions between the adhesives and PLA take place. It is for this reason that PC60 was taken forward for further investigation in the experimental investigation.

\section{EXPERIMENTAL MATERIALS AND METHODS}

\section{n-CA Polymerization Rate (Pull off Test)}

As widely reported, the curing process of CA-based adhesives is facilitated by the presence of moisture and occurs almost instantly at ambient temperatures (Klemarczyk, 2002). Simple and easily prepared cement mortar specimens with a thin bond line joint geometry were cast and then tested via a tensile (pull-off) test to evaluate the curing time of the trialled n-CA formulations. $5 \mathrm{~mm}$ diameter $30 \mathrm{~mm}$ long steel bolts were cast into the mortar samples (25 mm side cubes) with an embedment depth of $10 \mathrm{~mm}$, allowing the specimens to be gripped by the machine jaws, as shown in Figure 1.

Once the adhesive formulations had sufficiently cured, the samples were pulled apart at a rate of $0.05 \mathrm{~mm} / \mathrm{s}$, using a servo-hydraulic universal machine with a load cell capacity of $100 \mathrm{kN}$, and observations made regarding their modes of failure. The testing arrangement and a sample under testing can be seen in Figures 2A,B respectively.

\section{Cement Mortar Components and Curing Regime}

The mix constituents and proportions of the mortar comprised CEM II/A-L1 32,5R cement $\left(562 \mathrm{~kg} / \mathrm{m}^{3}\right) ; 0-2 \mathrm{~mm}$ dried fine sand $\left(1,124 \mathrm{~kg} / \mathrm{m}^{3}\right)$ and water $\left(253 \mathrm{~kg} / \mathrm{m}^{3}\right)$. In order to prepare fully dried surfaces, cement mortar samples were cured in a water tank for 4 days, dried in the oven for 2 days at $80^{\circ} \mathrm{C}$ and finally placed in the desiccator (with silica gel $\mathrm{RH}=30 \%$, temp $20^{\circ} \mathrm{C}$ ) for 1 day. Compression tests were conducted on three $50 \mathrm{~mm}$ cubes at 7,14 , 21 and 28. The tests were performed under load control using a calibrated Controls Automax 50-C52D02 machine at a rate of $1.2 \mathrm{MPa} / \mathrm{sec}$. The average compressive strength at 28 days was 42.7 $\mathrm{MPa}(\mathrm{CoV} 2 \%)$. For the sake of completeness, the variation of the cement mortar compressive strength with time is represented in Figure 3.

The surfaces were lightly sanded in order to reduce the influence of the demoulding oil and to remove any geometric irregularities. A thin layer of adhesive was placed on one side of the cubes using a $1 \mathrm{ml}$ plastic pipette, the average quantity of adhesive was $0.103 \mathrm{~g}$ ( $\mathrm{CoV}$ 9\%). The only load applied to the vertically arranged block assembly prior to testing was the selfweight of the upper block. The bond strength was calculated from the maximum force during the tension test carried to rupture and the contact cross-sectional area of the specimens (See Experimental Results, Tensile Test (Pull-Off)).

\section{Healing Agent}

The commercial Ethyl Cyanoacrylate - Procure PC60 - was chosen as the CA component (see n-CA Longevity Within $3 D$ Printed Polylactide Acid Capsules). The properties of the CA, as reported in the datasheet, are given in Table 5.

Four formulations containing a commercial monomer 2-ethyl cyanoacrylate (CA), acrylic acid (AA) and nitro-anthraquinone (nAq) were prepared by mixing the components CA:AC:nAq in the ratios $\mathrm{x}: 1: 0.02$, where $\mathrm{x}$ is in the range from 5 to 1 . All the formulations were prepared at room temperature, by the slow addition of the $\mathrm{AA}$ and the nAq at the prescribed concentrations, to the commercial CA adhesive. To facilitate the mixing of the chemical 

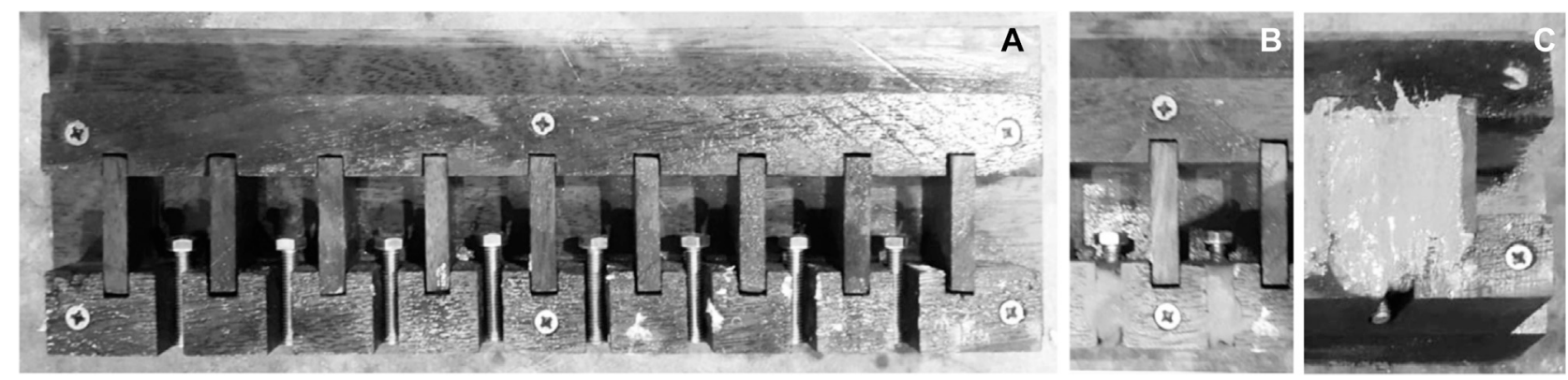

FIGURE 1 | Wooden cube mold $25 \mathrm{~mm}$ (A), steel bolts placed and sealed with the plasticine (B), sample after casting (C).

\section{A To force measurements}

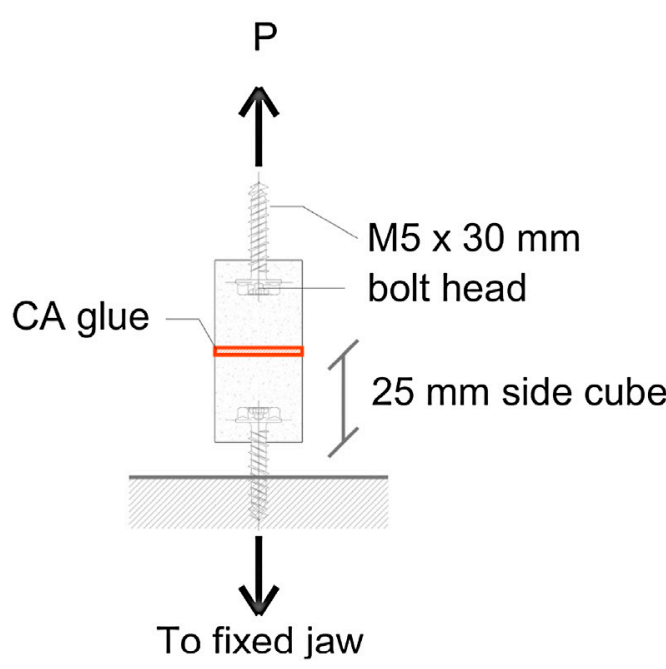

B

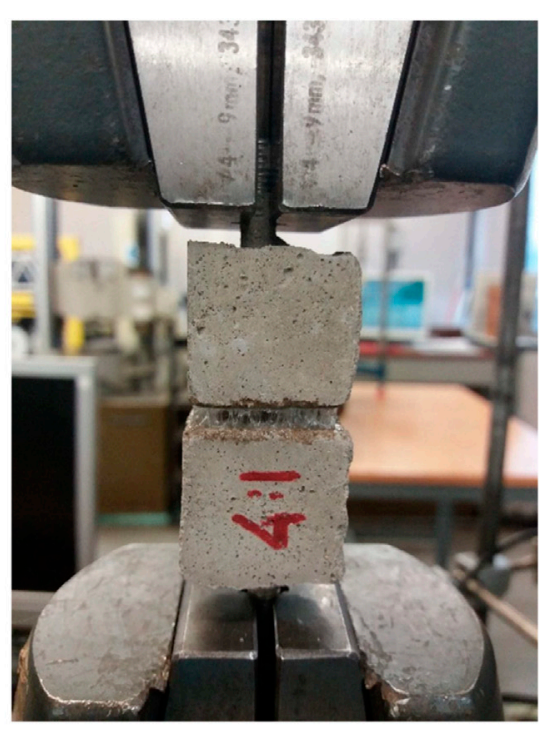

FIGURE 2 | Setup of the tensile strength test (A), sample under testing (B).

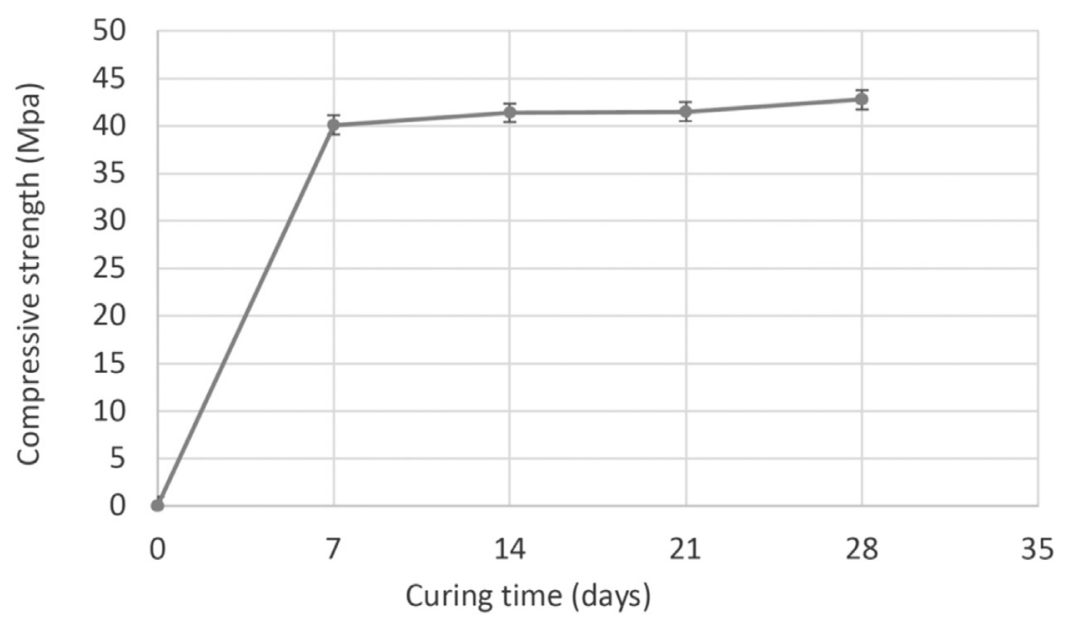

FIGURE 3 | Cement mortar compressive strength variation with time. 
TABLE 5 | CA properties.

\begin{tabular}{|c|c|c|c|c|c|c|c|}
\hline Chemical type & Appearance & Specific gravity & Shelf life $\left(5^{\circ} \mathrm{C}\right)$ & Viscosity $\left(\mathrm{cPs}^{1}\right)$ & Fixture time (secs) & Full cure (hours) & $\mathrm{ft}_{\mathrm{CA}}\left(\mathrm{N} / \mathrm{mm}^{2}\right)$ \\
\hline Alkoxy ethyl & Clear & 1.06 & 6 months & 100 & $10-50$ & 24 & 18 \\
\hline
\end{tabular}

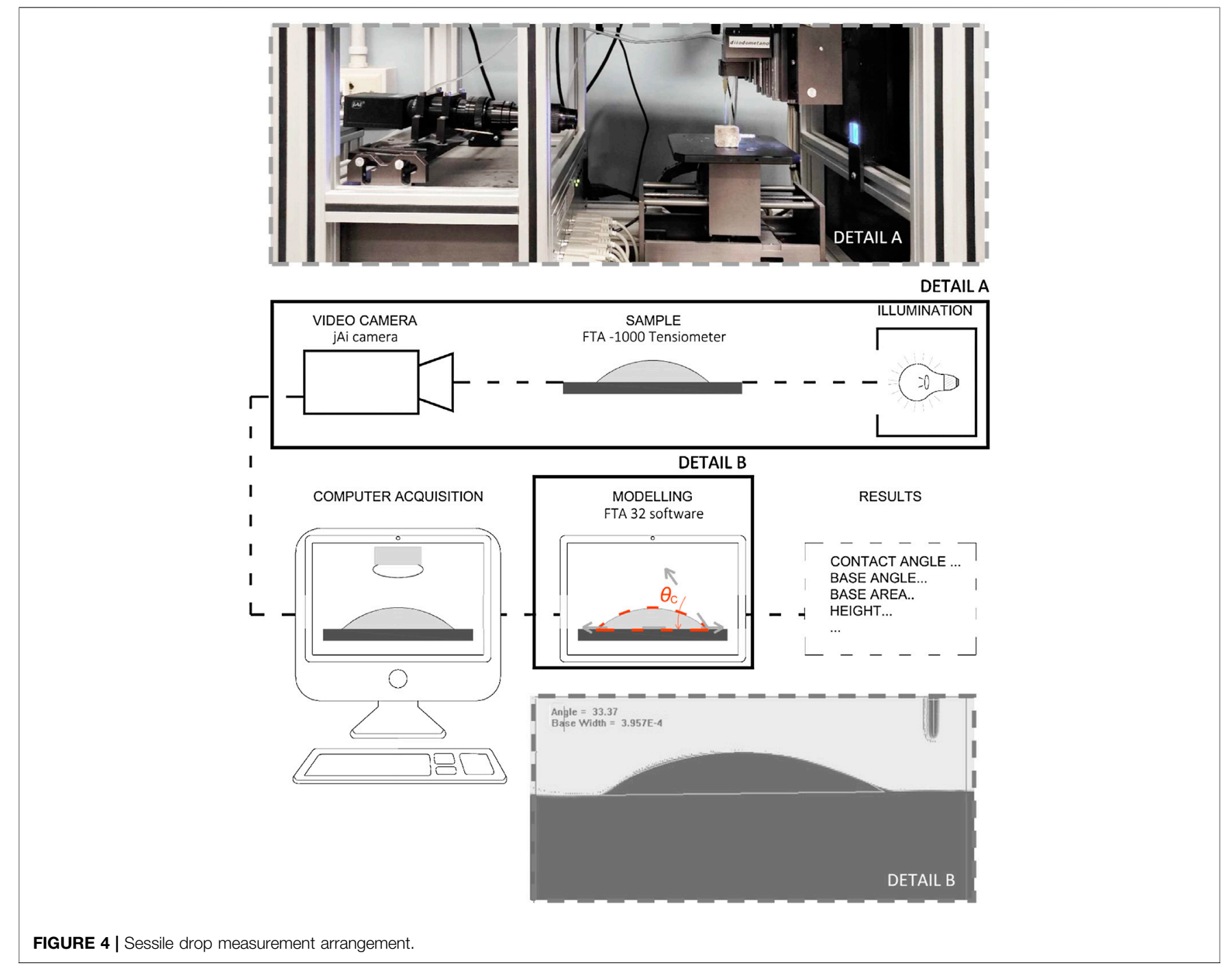

compounds, a polyurethane small tube was used to gently stir the mixture. A thin layer of adhesive was placed on one side of the cubes using a $1 \mathrm{ml}$ plastic pipette, the average quantity of adhesive was $0.103 \mathrm{~g}$ (CoV 9\%). The only load applied to the vertically arranged block assembly prior to testing was the self-weight of the upper block. The bond strength was calculated from the maximum force during the tension test carried to rupture and the contact cross-sectional area of the specimens (See section 4.2).

\section{Substrate-Contact Angle}

The influence of the CA/AA proportion in the four formulations on the contact angle $(\theta c)$ was examined via the sessile drop technique. The substrate was the open cast face of a cement mortar cube manufactured as described above for the pull-off test. The sessile drop technique, a schematic of which is shown in Figure 4, was performed using a drop volume of $10 \mu \mathrm{L}$. The technique was performed three times.

All the formulations were prepared immediately before the tests, which were performed under laboratory environmental conditions (i.e., untreated air at $20^{\circ} \mathrm{C}$ ) using a FTA $-1,000$ Tensiometer (First Ten Angstroms Inc., Portsmouth, Virginia, US). Each static contact angle was captured using a video with a jAi camera (jAi, Copenhagen, Denmark), and the angle measured from the image using FTA 32 software (First Ten Angstroms). Figure 4 shows the sessile drop measurement arrangement, in which detail A and B shows the tensiometer setup and a typical microscope image of 
TABLE 6 | Details of the experimental program.

\begin{tabular}{|c|c|c|c|c|c|c|c|c|}
\hline & \multirow[t]{3}{*}{ Sample designation } & \multirow[t]{3}{*}{ Ratio CA:AA:nAQ } & \multicolumn{6}{|c|}{ Tensile test (pull-off) specimen numbers } \\
\hline & & & \multicolumn{6}{|c|}{ Curing duration (days) } \\
\hline & & & 1 & 3 & 7 & 14 & & 21 \\
\hline \multirow[t]{7}{*}{ MECHANICAL TESTS } & 1_0/0_M (control) & 1:0:0 & 3 & - & - & - & & - \\
\hline & 1_5/1_m & $5: 1: 0.02$ & 3 & 3 & 3 & 3 & & 3 \\
\hline & 1_4/1_m & 4:1:0.02 & 3 & 3 & 3 & 3 & & 3 \\
\hline & 1_2/1_m & $2: 1: 0.02$ & 3 & 3 & 3 & 3 & & 3 \\
\hline & 1_1/1_m & $1: 1: 0.02$ & 3 & 3 & 3 & 3 & & 3 \\
\hline & & & \multicolumn{6}{|c|}{ Specimen numbers } \\
\hline & & & \multicolumn{2}{|l|}{$\begin{array}{c}\text { CONTACT } \\
\text { ANGLE }\end{array}$} & DSC/TGA & & NMR & \\
\hline \multirow[t]{5}{*}{ CHEMICAL-PHYSICAL TESTS } & 2_0/0_p (control) & 1:0:0 & 3 & & 1 & & 1 & \\
\hline & 2_5/1_p & $5: 1: 0.02$ & 3 & & 1 & & 1 & \\
\hline & 2_4/1_p & 4:1:0.02 & 3 & & 1 & & 1 & \\
\hline & 2_2/1_p & $2: 1: 0.02$ & 3 & & 1 & & 1 & \\
\hline & 2_1/1_p & $1: 1: 0.02$ & 3 & & 1 & & 1 & \\
\hline
\end{tabular}

sessile drop with the baseline and drop profile modeled by the software (light gray lines) respectively.

\section{Differential Scanning Calorimetry and Thermogravimetric Analysis}

It's well known that the $3 \mathrm{D}$ printing process might change the properties of the PLA spool material because of the effect of the heat treatment (Cuiffo et al., 2017). The extrusion process might change the glass transition temperature ( $\mathrm{Tg})$, melting temperature ( $\mathrm{Tm})$, etc and thus influencing the polymer properties. The chemical composition and structure of the PLA after 3D printing was assessed to ensure that it was compatible with the healing agent formulations. This assessment employed differential scanning calorimetry analyses (DSC), carried out on PLA samples collected directly from the roll and from a 3D printed TETs. TETs were printed from a commercial clear PLA (provided by Innofil 3D-BASF, $1.75 \mathrm{~mm}$ diameter) using an Ultimaker $2{ }^{\circledR}$ printer (Utrecht, Netherlands) with a $0.25 \mathrm{~mm}$ nozzle. More detailed information on the design and manufacture of the TETs can be found in De Nardi et al. (2020). Moreover, with the aim to characterize the main properties of the proposed formulations, thermogravimetric analysis (TGA). and differential scanning calorimetry (DSC) analyses were carried out on polymer samples exposed to laboratory air for 1 week of curing.

DSC and TGA analyses were conducted in a Linseis PTA ST1000 under nitrogen flow of $100 \mathrm{ml} \mathrm{min}^{-1}$ with a temperature ramp of $10^{\circ} \mathrm{C} \mathrm{min}{ }^{-1}$ from 30 to $250^{\circ} \mathrm{C}$ or $400^{\circ} \mathrm{C}$ depending on the type of measurement and polymer.

\section{Nuclear Magnetic Resonance spectroscopy}

In order to characterize the $\mathrm{n}$-AC polymers and to better understand the interaction between $\mathrm{CA}$ and $\mathrm{AA},{ }^{1} \mathrm{H} \mathrm{NMR}$ measurements were performed at room temperature on a Brucker Avance 400 II spectrometer operating at $400 \mathrm{MHz}$, using deuterated chloroform as a solvent. The inherently low sensitivity of DSC, TGA and NMR techniques only allows the verification of relatively high proportions of different species in the polymer (at least 2-5\%). For this reason, the n-CA formulation of 1:1 by weight of CA/AA was employed to help amplify the differences between the pure CA and the proposed formulations.

\section{EXPERIMENTAL PROGRAMME}

\section{Summary of Tests}

The experimental program comprised two groups of tests: 1) mechanical tests; 2) physical-chemical tests. The aim of Group 1 was to evaluate the curing time and the bond strength of all the trialled formulations. Tensile tests (pull-off) were carried out on cement mortar samples with a thin modified n-CA joint. The aim of Group 2, which comprises the substrate contact angle, Thermogravimetric analysis (TGA) and differential scanning calorimetry (DSC) and the nuclear magnetic resonance (NMR) analysis, is useful to characterize the main properties of the polymers Multiple tests series were undertaken in each of these groups, each of which had a different tested formulation, as summarized in Table 6.

\section{EXPERIMENTAL RESULTS}

\section{Tensile Test (Pull-Off)}

The average bond strengths calculated from the maximum force during the tension test and the bonded cross-sectional area of the mortar cubes are summarized in Table 7.

In all the samples bonded with the unmodified commercial CA (control specimens), failure occurred in the concrete sample at the level of the bolt head (See Figure 5A. This suggests that the bonded material had a higher tensile strength than the mortar. 
TABLE 7 | Pull off test results performed on samples with sanded surfaces.

\begin{tabular}{|c|c|c|c|c|c|}
\hline \multirow[t]{2}{*}{ Designation } & \multicolumn{5}{|c|}{ Curing time (days) } \\
\hline & 1 & 3 & 7 & 14 & 21 \\
\hline & Max stress (MPa) (CoV\%) & Max stress (MPa) (CoV\%) & Max stress (MPa) (CoV\%) & Max stress (MPa) (CoV\%) & Max stress (MPa) (CoV\%) \\
\hline 1_Control & $2.71(17)^{\mathrm{a}}$ & - & - & - & - \\
\hline 1_5/1 & Uncured & Uncured & $0.4(4)$ & $0.8(78)$ & $0.8(7)$ \\
\hline 1_4/1 & Uncured & Uncured & $0.5(27)$ & $0.7(8)$ & $2.6(50)$ \\
\hline 1_2/1 & Uncured & Uncured & Uncured & $0.5(26)$ & $1.0(72)$ \\
\hline 1_1/1 & Uncured & Uncured & Uncured & Uncured & $0.7(38)$ \\
\hline
\end{tabular}

${ }^{a}$ Tests failed in the concrete sample at the level of the bolt head.

Failure occurred at the bonded surface in all remaining tests that employed n-CA formulations. The addition of the AA, irrespective of the proportion, appears to have retarded the polymerization reactions by at least three days. Before this time none of the n-CA formulations had cured sufficiently to allow meaningful bond strengths to be determined. Moreover, a general reduction in bond stress occurs with the addition of AA. As can be seen in Figure 5B, a significant percentage of the n-CA applied to the surfaces appears to have been absorbed into the sample with only a proportion of the cross-section being fully bonded. The proportion of area bonded (b) was measured after each test using digital images of the failed surfaces. This proportion was used to calculate a bonded area (bA) and a bond strength $(p /(\mathrm{bA}))$, noting that $\mathrm{A}$ is the total cross-section $\left(625 \mathrm{~mm}^{2}\right)$. The average value of $b$ was 0.3 .

As summarized in Table 7, after 7 days the formulations designated as 5:1 and 4:1 showed a bond strength of 0.4 and $0.5 \mathrm{MPa}$ respectively; after 14 days the formulation 5:1 showed a bond strength doubled $(0.8 \mathrm{MPa})$, while in samples containing the $4: 1$ formulation the bond strength increase is $26 \%(0.7 \mathrm{MPa})$. After 21 days, the two formulations appeared to have a completely different behavior: the formulation 5:1 had a bond strength of $0.8 \mathrm{MPa}$, seemingly approaching a ceiling value. The formulation 4:1 exhibited a bond strength of $2.6 \mathrm{MPa}$, with a significantly increasing rate over the duration considered.

The formulations designated as 2:1 and 1:1 required 14 and 21 days respectively to obtain a suitably cured state. The samples glued with the formulation 2:1 doubled the bond strength after 21 days $(1.0 \mathrm{MPa})$ with respect to the value obtained at 14 days $(0.5 \mathrm{MPa})$, showing a constant increasing cure rate. As expected, the 1:1 formulation which contained the greatest quantity of retarder among the trialled formulations, exhibited the slowest curing speed. Visual observations revealed the appearance of a partially cured layer of adhesive after 21 days with an associated bond strength of $0.7 \mathrm{MPa}$. A comparison of the pull-off test results - which strongly depend on the adhesive strength to the substrate, internal cohesive strength, and a reasonable curing time-showed that the $4: 1$ formulation had the highest bonding characteristics over time.

The variability of the test results, as indicated by the standard deviation, is due to various factors inherent to the testing
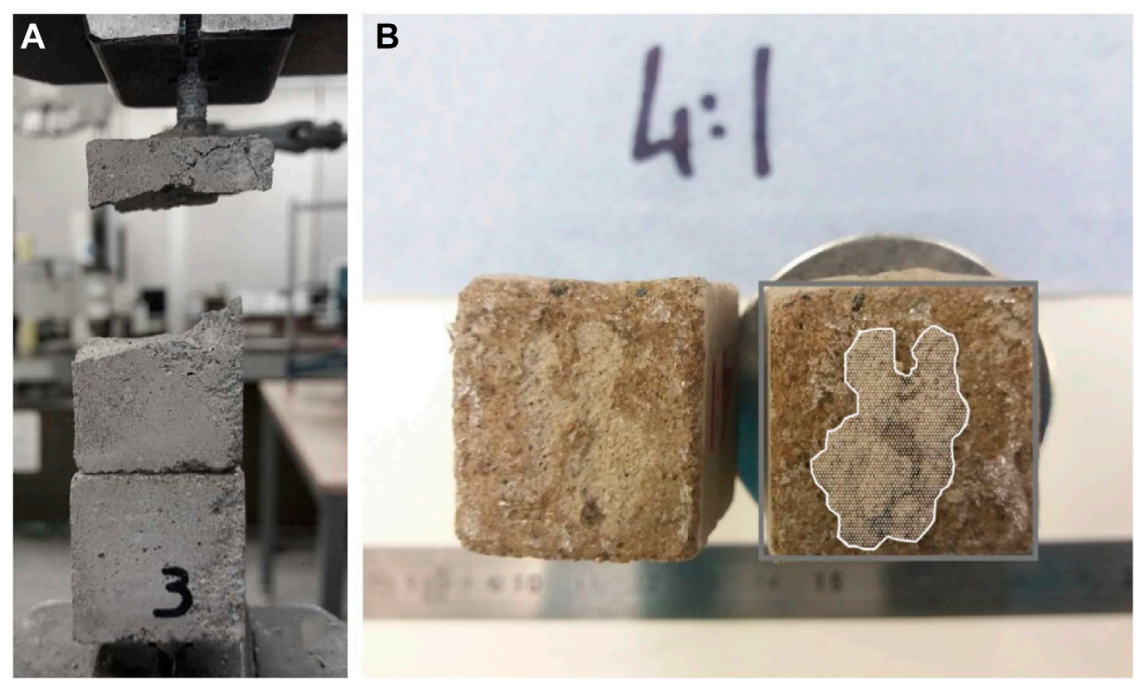

FIGURE 5 | Sample bonded with the unmodified commercial CA: failure occurred in the concrete sample at the level of the bolt head (A); bonded cross -section of the samples after testing (B). 
procedure when inhomogeneous substrates are studied (Croll et al., 2014; Mareš et al., 2014; Sogutlu, 2017). It is also reasonable to assume that small-scale samples might result in a greater variability, due to possible geometrical irregularities such as non-perfect face coplanarity.

\section{Substrate Contact Angle}

The contact angle reported for cement mortar substrates was taken as the average contact angle of the three drops on that substrate and is provided in Table 8 along with the coefficients of variation (COV\%).

For all the n-CA formulations analyzed, the contact angle (as represented in Table 4 (c)) was smaller than the commercial CA monomer, suggesting a higher degree of wettability. It may be reasonably assumed that the addition of the solvent, such as the AA, decreases the viscosity of the compounds and subsequently increases the wettability. Moreover, the well-known AA hydrophilicity (Akbari et al., 2017) might affect the contact angle in different steps of the polymerization reaction, resulting in changes of the substrate/adhesive interface. As observed, a significant decrease of the contact angle can be achieved by increasing the acrylic acid content. The contact angle of the 5:1

TABLE 8 | Contact angle results for varying $n-C A$ formulations.
Designation n-CA formulations

2_1:0 - pure PC60

2_5:1

2_4:1

2_2:1

2_1:1
Contact angle $\theta \mathrm{c}$ (degrees) (CoV\%)

26 (18)

22 (35)

11 (17)

11 (16)

6 (38) formulation is slightly smaller than the pure CA, featuring a decrease which is proportional to the AA content ( $17 \%)$. Interestingly the 4:1 and 2:1 formulation showed a similar contact angle value $\left(11^{\circ}\right)$, suggesting an increase of $60 \%$ of the wettability to the substrate surface with respect to the pure CA.

The 1:1 formulation, featured an increase in wettability of $75 \%\left(6^{\circ}\right)$.

\section{Polymer Characterization: Differential Scanning Calorimetry/Thermogravimetric Analysis and Nuclear Magnetic Resonance Analysis}

Figure 6A presents the DSC results of the clear PLA used to manufacture the TETs. The results show that the filament collected from the roll and the polymer collected after the $3 \mathrm{D}$ printing process do not show substantial differences in the thermogram, with the glass transition $\left(54^{\circ} \mathrm{C}\right)$ and the crystallization peak $\left(123^{\circ} \mathrm{C}\right)$ temperatures being the same. The melting point of the $3 \mathrm{D}$ printed polymer was found to be $3^{\circ} \mathrm{C}$ lower than that of the filament and in both cases exothermic crystallization peaks can be found at $123^{\circ} \mathrm{C}$, which is typical of partially amorphous PLA (Cuiffo et al., 2017). This suggests that no significant changes in chemistry had occurred as result of the $3 \mathrm{D}$ printing process; despite the thermal cycles related to the TETs 3D printing process, there is no evidence of polymer degradation.

The material does not decompose until $250^{\circ} \mathrm{C}$ and no weight loss can be attributed to chemical reaction and or solvent loss.

Figure 6B shows the thermogram of the pure CA and the 1:1 formulation. The measurements highlighted that the two polymers are quite different, the pure CA is rigid, white and translucent, whilst the 1:1 polymer appeared transparent and quite soft. The thermograms are quite similar, the pure CA shows
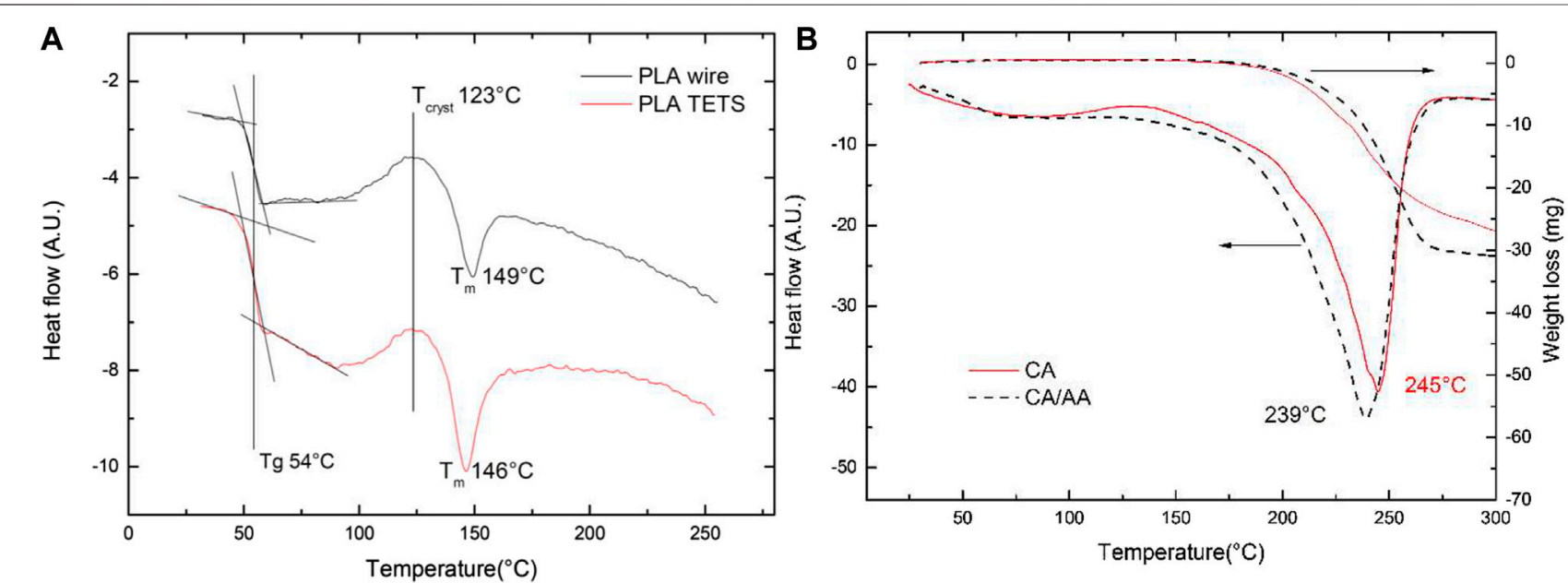

FIGURE 6 | Comparison of the DSC of PLA employed in the making of the TETS. Run Conditions $\mathrm{N} 2 \mathrm{flow} 100 \mathrm{ml}$ min-1, temperature ramp $10^{\circ} \mathrm{C}$ min-1 (A) Comparison of the DSC-TGA of the polymerized CA and CA/A adhesives after a week of curing in air. Run Conditions $\mathrm{N} 2 \mathrm{flow} 100 \mathrm{ml}$ min-1, temperature ramp $10^{\circ} \mathrm{C}$ min-1 (B). 


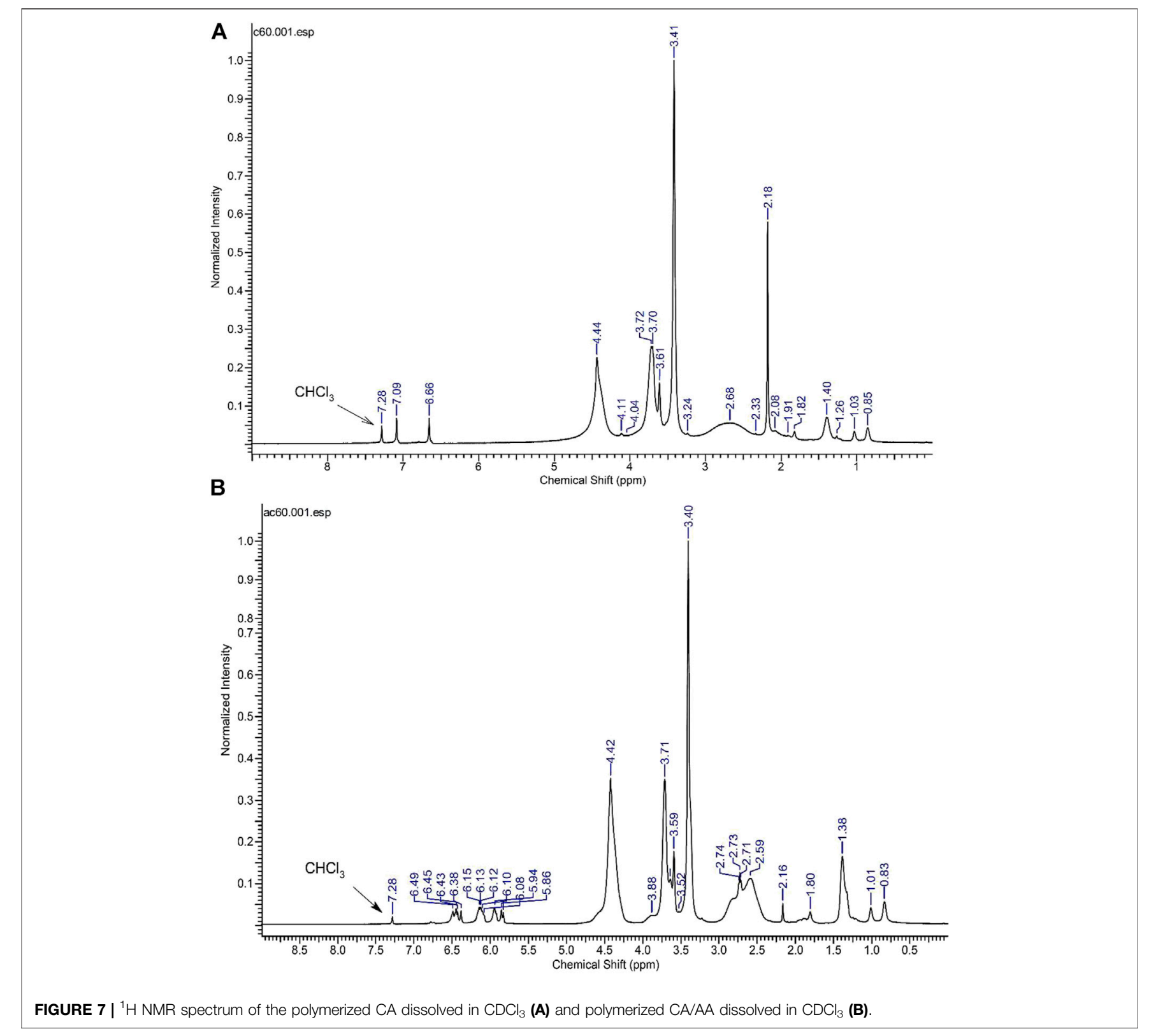

a peak of decomposition at $245^{\circ} \mathrm{C}$, whilst the $1: 1$ polymer shows a displacement of this peak to $239^{\circ} \mathrm{C}$. There is no weight loss for the 1:1 formulation in the region of the boiling point of the AA $\left(141^{\circ} \mathrm{C}\right)$, thus suggesting a strong interaction of the AA with the pure CA.

The pure CA polymerization mechanism follows that of anionic polymerization. In the case of a mixture containing $\mathrm{CA}, \mathrm{AA}$ and $\mathrm{nAq}$, it can be reasonably assumed that AA may polymerize by a slow thermal-radical process, which is inhibited by the presence of hydroquinone. However, the nature of the resulting polymer requires further investigation"

The ${ }^{1} \mathrm{H}$ NMR spectra of the commercial CA is reported in Figure 7A. Several signals can be seen, which suggests a formulation comprising different monomers. Moreover, the presence of the peaks at 7.09 and 6.66 ppm suggests two vinyl protons probably due to free monomers. The NMR spectrum of the 1:1 formulation dissolved in deuterated chloroform, shown in Figure $\mathbf{7 B}$, shows some additional peaks. The presence of non-polymerized monomers can by deduced by assigning, in the region $5.86-6.49 \mathrm{ppm}$, to vinyl protons of various monomers. The presence of new peaks, and the broadening and change of intensity of others suggests the formation of several polymeric species; however, the study of the polymer nature is beyond the scope of the present work. In summary, the addition of $\mathrm{AA}$ to the commercial monomer $\mathrm{CA}$ appears to modify 
both the macroscopic and molecular properties of the resulting polymer.

\section{CONCLUSION}

In this research, the properties of innovative cyanoacrylate adhesives ( $\mathrm{n}-\mathrm{CA}$ ) have been investigated for applications in self-healing concrete technologies. The study explored the properties of newly designed formulations, which involved the additions of retarders such as the acrylic acid (AA) and the nitro-anthraquinone ( $\mathrm{n}-\mathrm{AQ}$ ) in different ratios to a commercial cyanoacrylate (CA) adhesive.

The design of the $\mathrm{n}$-CA formulation focused first on reducing the curing time. This was accomplished by introducing components that were chemically compatible with the PLA of the 3D printed minivascular networks (MVNs) units. It was also important that the n-CA maintained good bond properties with cementitious substrates.

From the analyses of the results presented in the paper, the following main conclusions have been drawn:

- The n-CA formulations form the basis for an innovative healing agent able to act efficiently and with good encapsulation stability. Among the retarder candidates studied, the addition of both AA and $\mathrm{n}-\mathrm{AQ}$ to a commercial CA product resulted in the formation of compounds with an ability to remain liquid within PLA carriers for longer durations than commercial CA adhesives alone.

- The polymerization rate and the bond strength to the cementitious matrix of four formulations (CA:AA:nAq in the ratios 5-1:1:0.02) were evaluated. All of the CA formulations exhibited a significant increase in curing time with respect to the unmodified CA, whilst in the best case (4:1) the tensile bond strength only decreased by $4 \%$ less. Nevertheless, the resultant values remain compatible with the mechanical properties of the cement matrix. By increasing the proportion of the retarder, the wettability was significantly improved.

- The 4:1 formulation showed the highest bond strength over time, and good wettability (contact angle) to cementitious substrates. It showed a significant increase in bond strength from 14 days to 21 , with a ceiling value of $2.6 \mathrm{MPa}$.

- The greater absorption of the n-CA formulations into the cementitious matrix over the curing period, compared with the reference CA, implies that it is important to ensure an adequate supply of $n-C A$ for the full healing potential of the $\mathrm{n}$-CA formulations to be realised.

- TGA analysis carried out on PLA samples highlighted that no relevant changes in chemistry occurred as a result of the $3 \mathrm{D}$ printing process. TGA/DSC analysis showed a chemical interaction between the $\mathrm{CA}$ and $\mathrm{AA}$, with the formation of a new polymer, completely different in nature from the commercial CA monomer. NMR spectroscopy confirmed this hypothesis.
It has been demonstrated that it is possible to manipulate the properties of a commercial adhesive to begin to satisfy the various requirements for a healing agent in an encapsulated self-healing system. The next phase of research will explore the crack healing potential and the enhanced durability performance of concrete structures containing vascular and mini-vascular networks with modified CA-based healing agents. To enable this, studies are needed to optimize the CA formulation via mix design, refine the polymerization mechanism and examine the mechanical properties of the healing system under a range of environmental conditions. This, in turn, will allow self-healing systems to be further refined and optimized for targeted healing of varying spatial and/or temporal damage, which will ultimately facilitate the adoption of self-healing materials by the construction industry.

\section{DATA AVAILABILITY STATEMENT}

The datasets presented in this study can be found in online repositories. The names of the repository/repositories and accession number(s) can be found below: Cardiff University data catalogue at 10.17035/d.2021.0128548280.

\section{AUTHOR CONTRIBUTIONS}

Conceptualization CN, DG, LR and T); Methodology, CN, LR; Formal analysis, $\mathrm{CN}$, Writing-original draft preparation, Writing-review and editing, DG, TJ, and CN Investigation: CN (pull-off), LR, AV (TGA/DSC, NMR), GC (solubility test), DC (contact angle); Supervision, TJ and DG; Project administration, TJ; Funding acquisition, TJ and DG. All authors have read and agreed to the published version of the manuscript.

\section{FUNDING}

This research was funded by the Engineering and Physical Sciences Research Council (EPSRC), grant number EP/P02081X/1.

\section{ACKNOWLEDGMENTS}

The authors would also like to acknowledge the considerable help and expertize of Richard Thomas and Carl Wadsworth without whom the laboratory work would not have been possible. Thanks also to Marco Bortoluzzi for the laboratory support.

\section{SUPPLEMENTARY MATERIAL}

The Supplementary Material for this article can be found online at: https://www.frontiersin.org/articles/10.3389/fbuil.2021.660562/ full\#supplementary-material 


\section{REFERENCES}

Akbari, S., Eslahi, N., and Kish, M. H. (2017). Evaluation of Hydrophilic Properties of Acrylonitrile/acrylic Acid Copolymer Films Dendrigrafted with Citric Acid. Polyolefins J. 4 (2). doi:10.22063/poj.2017.1436

Croll, C., Siripirom, C., and Keil, B. (2014). Pull-Off Adhesion Test for Coatings on Large Pipes: Possible Variations in Failure Location and Mode. Forefr. Innov. Sustain., 2014. 1319-1333. doi:10.1061/9780784413692.119

Cuiffo, M., Snyder, J., Elliott, A., Romero, N., Kannan, S., and Halada, G. (2017). Impact of the Fused Deposition ( FDM ) Printing Process on Polylactic Acid (PLA) Chemistry and Structure. Appl. Sci. 77 (6), 579. doi:10.3390/app7060579

Davies, R. , Jefferson, A., Robert, L., and Gardner, D. (2015). A Novel 2d Vascular Network in Cementitious Materials. FIB Proc. 190. 108572.

Davies, R., Jefferson, T., and Gardner, D. (2021). Development and Testing of Vascular Networks for Self-Healing Cementitious Materials. J. Mater. Civ. Eng. 33 (7), 4021164. doi:10.1061/(ASCE)MT.1943-5533.0003802

De Nardi, C., Gardner, D., and Jefferson, A. D. (2020). Development of 3D Printed Networks in Self-Healing Concrete," Materials 13 (6), 1328. doi:10.3390/ ma13061328

Dry, C. (1994). Matrix Cracking Repair and Filling Using Active and Passive Modes for Smart Timed Release of Chemicals from Fibers into Cement matrice.Pdf. Smart Mater. Struc 3, 118-123. doi:10.1088/0964-1726/3/2/006

Ebnesajjad, S., Ed., (2009). "Characteristics of Adhesive Materials," In Adhesives Technology Handbook, Second Edi., Norwich, NY: William Andrew Publishing, pp. 63-135.

First Ten Angstroms. DCA 100 catalog. Available at: https://www. firsttenangstroms.com/dca-100-tensiometer (Accessed December 23, 2020).

Gardner, D., Herbert, D., Jayaprakash, M., Jefferson, A., and Paul, A. (2017). Capillary Flow Characteristics of an Autogenic and Autonomic Healing Agent for Self-Healing Concrete. J. Mater. Civ. Eng.29 (11), 04017228. doi:10.1061/ (asce)mt.1943-5533.0002092

Gardner, D., Jefferson, A., and Hoffman, A. (2012). Investigation of capillary flow in discrete cracks in cementitious materials. Cem. Concr. Res.42 (7), 972 -981. doi:10.1016/j.cemconres.2012.03.017

Hirozo, M., Yoshio, K., Tomoya, N., and Koji, O. (2000). Fundamental Study on Development of Intelligent Concrete Characterized by Self-Healing Capability for Strength. Concr. Res. Technol. 11 (2), 21-28. doi:10.3151/crt1990.11.2_21

Joseph, C., Gardner, D., Jefferson, T., Isaacs, B., and Lark, B. (2011). Self-healing Cementitious Materials: a Review of Recent Work. Proc. Inst. Civ. Eng. - Constr. Mater. 164 (1), 29-41. doi:10.1680/coma.900051

Joseph, C., Jefferson, A. D., and Cantoni, M. B. (2007). Issues Relating To the Autonomic Healing of Cementitious Materials.First Int. Conf. Self Heal. Mater.1 -8.

Joseph, C., Jefferson, A. D., Isaacs, B., Lark, R., and Gardner, D. (2010). Experimental Investigation of Adhesive-Based Self-Healing of Cementitious Materials. Mag. Concrete Res. 11, 831-843. doi:10.1680/MACR.2010.62.11.831

Klemarczyk, P. (2002). "Cyanoacrylateinstant Adhesives," in In Adhesion Science And Engineering. Editors A. V. Pocius and D. A. Dillard. (Amsterdam, Netherlands: Elsevier Science V.V)

Klemarczyk, P., and Guthrie, J. (2010). "5 - Advances in Anaerobic and Cyanoacrylate Adhesives," in In Woodhead Publishing Series In Welding And Other Joining Technologies, D. A. B. T.-A. Editor S. A. B. Dillard (New Delhi, Delhi: Woodhead Publishing), 96-131.
Kotzev, D. L., Ward, T. C., and Dwight, D. W. (1981). Assessment of the Adhesive Bond Properties of Allyl 2-cyanoacrylate. J. Appl. Polym. Sci. 26 (6), 1941-1949. doi:10.1002/app.1981.070260618

Li, V. C., Lim, Y. M., Chan, Y. W., Mook, Y., and Chan, Y. W. (1998). Feasibility Study of a Passive Smart Self-Healing Cementitious Composite. Compos. Part. B Eng. 29 (6), 819-827. doi:10.1016/S1359-8368(98)00034-1

Nishiwaki, T., Mihashi, H., Jang, B., and Miura, K. (2014). Development of Self-Healing System for Concrete with Selective Heating Around Crack Development of SelfHealing System for Concrete with Selective Heating Around Crack. J. Struct. Construction Eng. (Transactions AIJ) 70 (593), 25-30. doi:10.3130/aijs.70.25_3

Odian, G. (2004). Principles of Polymerization, 4th Ed, New York: John Wiley \& Sons,

Petrie, E. M. (2007). Handbook of Adhesives and Sealants. Second Edition2nd ed. New York: McGraw-Hill Education.

Quartarone, G., Ronchin, L., Tosetto, A., and Vavasori, A. (2014). New Insight on the Mechanism of the Catalytic Hydrogenation of Nitrobenzene to 4aminophenol in $\mathrm{CH} 3 \mathrm{CN}-\mathrm{H} 2 \mathrm{o}-\mathrm{Cf} 3 \mathrm{cooh}$ as a Reusable Solvent System. Hydrogenation of Nitrobenzene Catalyzed by Precious Metals Supported on Carbon. Appl. Catal. A. Gen. 475, 169-178. doi:10.1016/j.apcata.2014. 01.033

Selvarajoo, T., Davies, R. E., Freeman, B. L., and Jefferson, A. D. (2020). Mechanical Response of a Vascular Self-Healing Cementitious Material System under Varying Loading Conditions. Constr. Build. Mater. 254, 119245. doi:10. 1016/j.conbuildmat.2020.119245

Irving, S., (1990). “Adhesive Materials, Cyanoacrylate Adhesives," In Handbook of Adhesives, 3rd editio., New York: Chapman \& Hall.

Sogutlu, C. (2017). Determination of the Effect of Surface Roughness on the Bonding Strength of Wooden Materials. BioRes 12 (1), 1417-1429. doi:10. 15376/BIORES.12.1.1417-1429

Sun, L., Yu, W. Y., Ge, Q., Ling Sun, Q. G., and Wen, Yong. Yu. (2011). Experimental Research on the Self-Healing Performance of Micro-cracks in Concrete Bridge. Adv. Mater. Res. 250-253, 28-32. doi:10.1016/j.jmrt.2020. 04.053

Mareš, V., Štamborská, M., and Fajkoš, R. (2014). Comparison of the Adhesives Used in the Pull-Off Test Mo Layer of Railway Axle. Kovove Materialy 52 (3) 179-182. doi:10.4149/km_2014_3_179

Xue, C., Li, W., Castel, A., Wang, K., and Sheng, D. (2020). Effect of Incompatibility between Healing Agent and Cement Matrix on Self-Healing Performance of Intelligent Cementitious Composite. Smart Mater. Struct. 29, 115020. doi:10. 1088/1361-665X/aba9ac

Zhang, W., Zheng, Q., Ashour, A., and Han, B. (2020). Self-healing Cement Concrete Composites for Resilient Infrastructures: A Review. Compos. Part. B 189 (2), 107892. doi:10.1016/j.compositesb.2020.107892

Conflict of Interest: The authors declare that the research was conducted in the absence of any commercial or financial relationships that could be construed as a potential conflict of interest.

Copyright $\odot 2021$ De Nardi, Gardner, Cazzador, Cristofori, Ronchin, Vavasori and Jefferson. This is an open-access article distributed under the terms of the Creative Commons Attribution License (CC BY). The use, distribution or reproduction in other forums is permitted, provided the original author(s) and the copyright owner $(s)$ are credited and that the original publication in this journal is cited, in accordance with accepted academic practice. No use, distribution or reproduction is permitted which does not comply with these terms. 


\section{APPENDIX A}

\begin{tabular}{|c|c|c|c|c|}
\hline Compound & Formula & CAS \# & Company & Link to the datasheet \\
\hline Acrylic acid & $\begin{array}{c}\mathrm{CH}_{2}= \\
\mathrm{CHCOOH}\end{array}$ & $79-10-7$ & Sigma-aldrich & $\begin{array}{l}\text { https://www.sigmaaldrich.com/MSDS/MSDS/DisplayMSDSPage.do? } \\
\text { country=IT\&language=it\&productNumber=147230\&brand=ALDRICH\&PageToGoToURL=https\%3A } \\
\text { \%2F\%2Fwww.sigmaaldrich.com\%2Fcatalog\%2Fsearch\%3Fterm\%3D79-10-7\%26interface\% } \\
\text { 3DCAS\%2520No.\%26N\%3D0\%26mode\%3Dmatch\%2520partialmax\%26lang\%3Dit\%26region\% } \\
\text { 3DIT\%26focus\%3Dproduct }\end{array}$ \\
\hline Anthraquinone & $\mathrm{C}_{14} \mathrm{H}_{8} \mathrm{O}_{2}$ & $84-65-1$ & Sigma & $\begin{array}{l}\text { https://www.sigmaaldrich.com/MSDS/MSDS/DisplayMSDSPage.do? } \\
\text { country=IT\&language=it\&productNumber=A90004\&brand=ALDRICH\&PageToGoToURL=https\%3A } \\
\text { \%2F\%2Fwww.sigmaaldrich.com\%2Fcatalog\%2Fsearch\%3Fterm\%3D84-65-1\%26interface\% } \\
\text { 3DCAS\%2520No.\%26N\%3D0\%26mode\%3Dpartialmaxfocus\%3Dproduct\%26lang\%3Dit\% } \\
\text { 26region\%3DIT\%26focus\%3Dproduct\%26gclid\%3DCjwKCAjwpqv0BRABEiwA- } \\
\text { TySwFWuBNHr7wwJUhDAT1f85warSxY39IUJF9K5QDtPs1pGd1bYkhtlhoCYpoQAvD_BwE }\end{array}$ \\
\hline Chloroform & $\mathrm{CHCl}_{3}$ & $67-66-3$ & Sigma & $\begin{array}{l}\text { http://www.sigmaaldrich.com/MSDS/MSDS/DisplayMSDSPage.do? } \\
\text { country=IT\&language=it\&productNumber=C7559\&brand=SIGMA\&PageToGoToURL=http\%3A\%2F } \\
\% 2 \text { Fwww.sigmaaldrich.com\%2Fcatalog\%2Fproduct\%2Fsigma\%2Fc7559\%3Flang\%3Dit }\end{array}$ \\
\hline Dichloromethane & $\mathrm{CH}_{2} \mathrm{Cl}_{2}$ & $75-09-2$ & Sigma-aldrich & $\begin{array}{l}\text { http://www.sigmaaldrich.com/MSDS/MSDS/DisplayMSDSPage.do? } \\
\text { country=IT\&language=it\&productNumber=443484\&brand=SIAL\&PageToGoToURL=http\%3A\%2F } \\
\text { \%2Fwww.sigmaaldrich.com\%2Fcatalog\%2Fproduct\%2Fsial\%2F443484\%3Flang\%3Dit }\end{array}$ \\
\hline Diethyl ether & $\left(\mathrm{CH}_{3} \mathrm{CH}_{2}\right)_{2} \mathrm{O}$ & $60-29-7$ & Sigma-aldrich & $\begin{array}{l}\text { http://www.sigmaaldrich.com/MSDS/MSDS/DisplayMSDSPage.do? } \\
\text { country=IT\&language=it\&productNumber=346136\&brand=SIAL\&PageToGoToURL=http\%3A\%2F } \\
\text { \%2Fwww.sigmaaldrich.com\%2Fcatalog\%2Fproduct\%2Fsial\%2F346136\%3Flang\%3Dit }\end{array}$ \\
\hline Ethyl acrylate & $\begin{array}{c}\mathrm{CH}_{2}= \\
\mathrm{CHCOOOC}_{2} \mathrm{H}_{5}\end{array}$ & $140-88-5$ & Sigma-aldrich & $\begin{array}{l}\text { https://www.sigmaaldrich.com/MSDS/MSDS/DisplayMSDSPage.do? } \\
\text { country=IT\&language=it\&productNumber=W241811\&brand=ALDRICH\&PageToGoToURL=https\% } \\
\text { 3A\%2F\%2Fwww.sigmaaldrich.com\%2Fcatalog\%2Fsearch\%3Fterm\%3D140-88-5\%26interface\% } \\
\text { 3DCAS\%2520No.\%26N\%3D0\%26mode\%3Dpartialmaxfocus\%3Dproduct\%26lang\%3Dit\% } \\
\text { 26region\%3DIT\%26focus\%3Dproduct\%26gclid\%3DCjwKCAjwpqvOBRABEiwA- } \\
\text { TySwbU7Ojc31QkPorNXQcjbiKb8SNKLgJIzWWZcjvpcC4AvbLuakBCPahoC6BgQAvD_BwE }\end{array}$ \\
\hline Styrene & $\mathrm{C}_{6} \mathrm{H}_{5} \mathrm{CH}=\mathrm{CH}_{2}$ & $100-42-5$ & Aldrich & $\begin{array}{l}\text { http://www.sigmaaldrich.com/MSDS/MSDS/DisplayMSDSPage.do? } \\
\text { country=IT\&language=it\&productNumber=W323306\&brand=ALDRICH\&PageToGoToURL=http\% } \\
\text { 3A\%2F\%2Fwww.sigmaaldrich.com\%2Fcatalog\%2Fproduct\%2Faldrich\%2Fw323306\%3Flan }\end{array}$ \\
\hline $\begin{array}{l}\text { Trifluoroacetic } \\
\text { anhydride }\end{array}$ & $\mathrm{C}_{4} \mathrm{~F}_{6} \mathrm{O}_{3}$ & $407-25-0$ & Sigma-aldrich & $\begin{array}{l}\text { https://www.sigmaaldrich.com/MSDS/MSDS/DisplayMSDSPage.do? } \\
\text { country=IT\&language=it\&productNumber=106232\&brand=SIAL\&PageToGoToURL=https\%3A\%2F } \\
\text { \%2Fwww.sigmaaldrich.com\%2Fcatalog\%2Fsearch\%3Fterm\%3D407-25-0\%26interface\%3DCAS } \\
\text { \%2520No.\%26N\%3D0\%26mode\%3Dpartialmax\%26lang\%3Dit\%26region\%3DIT\%26focus\% } \\
\text { 3Dproduct }\end{array}$ \\
\hline
\end{tabular}




\section{APPENDIX B}

\section{List of abbreviations}

CA

$n-C A$

$n A Q$

$\mathrm{AA}$

MVNs

TETS

PLA

DSC

TGA

$\mathrm{Tg}$

Tm

NMR

SS

Ggbs

$\theta_{\mathrm{c}}$
Cyanoacrylate

Modified cyanoacrylate

Anthraquinone

Acrylic acid

Mini vascular networks

MVNs with a 3D tetrahedral shape

Polylactide acid

Differential scanning calorimetry

Thermogravimetric analysis

Glass transition temperature

Melting temperature

Nuclear magnetic resonance

Sodium silicate

Ground granulated blast-furnace slag

Contact angle 